[RAdiocarbon, Vol 26, No. 3, 1984, P 412-430]

\title{
QUEENS COLLEGE RADIOCARBON MEASUREMENTS IV
}

RICHARD R PARDI, LYNN TOMECEK, and WALTER S NEWMAN Radiocarbon Laboratory, Queens College, City University of New York, Flushing, New York 11367

The following list includes radiocarbon analyses of samples related to studies of Holocene sea levels completed since the publication of the last list ( $\mathrm{R}, 1980, \mathrm{v} 22$, p 1073-1083). Sample preparation and counting for liquid scintillation samples remain the same. However, an additional gasproportional facility was added in 1981 to handle the analyses of small samples, some of which are included in this list. The new system consists of two 660cc OFHC copper counters built at Queens College. Samples are counted over at least two 2800 minute intervals alternating with backgrounds and standards counted over 1400 minute intervals. Ages are based on the Libby half-life of 5568 years and include $1 \sigma$ standard deviations of sample, standard, and background activities.

\section{ACKNOWLEDGMENTS}

The authors thank M Newman and $\mathrm{K}$ Tessmer for their able assistance in the laboratory. This work was supported by grant EAR 77-13666 from the National Science Foundation, contract 14-08-0001-17729 with the United States Geological Survey and grant RF-11661 from the City University of New York.

New York

\section{Marlboro Marsh series}

This series was taken from Marlboro Marsh. All samples are basal peat, at coordinates $\left(41^{\circ} 36^{\prime} 40^{\prime \prime} \mathrm{N}, 73^{\circ} 57^{\prime} 58^{\prime \prime} \mathrm{W}\right)$, and coll 1979 by $\mathrm{J}$ Miller, H Craig and L J Cinquemani except where noted.

QC-341. Marlboro Marsh 1

$2330 \pm 240$

3.1 to $3.3 \mathrm{~m}$ below mean high water.

QC-340. Marlboro Marsh 2

$3010 \pm 120$

4.1 to $4.9 \mathrm{~m}$ below mean high water.

QC-342. Marlboro Marsh 3

$4150 \pm 100$

4.8 to $5 \mathrm{~m}$ below mean high water.

QC-343. Marlboro Marsh 4

$4390 \pm 220$

5.8 to $6 \mathrm{~m}$ below mean high water.

QC-705. Marlboro Marsh 5

$4260 \pm 130$

7.15 to $7.45 \mathrm{~m}$ below mean high water. Coll by $\mathrm{H}$ Craig, S Jencius, and J Wilson.

QC-686. Marlboro Marsh 6

$$
4570 \pm 110
$$

8.2 to $8.6 \mathrm{~m}$ below mean high water. Coll by W S Newman, H Craig, $\mathrm{S}$ Jencius, and J Wilson. Comment: Marlboro 5 and 6 are on stiff clayey substrates and may not be valid sea level indicators. 


\section{Constitution Island series}

This series was taken from Constitution I. Marsh. All samples are basal peat. Coordinates for Constitution I. marsh are: Constitution I. 2, 7, and $12\left(41^{\circ} 24^{\prime} 22^{\prime \prime} \mathrm{N}, 73^{\circ} 56^{\prime} 53^{\prime \prime} \mathrm{W}\right)$, Constitution I. 5, 8, 11, and 13 $\left(41^{\circ} 24^{\prime} 40^{\prime \prime} \mathrm{N}, 73^{\circ} 56^{\prime} 53^{\prime \prime} \mathrm{W}\right)$, and Constitution I. 1, 3, 4, 6, 9, 10, and 14 $\left(41^{\circ} 24^{\prime} 23^{\prime \prime} \mathrm{N}, 73^{\circ} 56^{\prime} 30^{\prime \prime} \mathrm{W}\right)$.

QC-691. Constitution I. 1

$2320 \pm 500$

1 to $1.3 \mathrm{~m}$ below mean high water. Coll 1979 by W S Newman, H Craig, S Jencius, and J Wilson. Comment: sample yields only freshwater diatoms; also some benzene evaporated.

QC-1039. Constitution I. 2

$2160 \pm 130$

1.7 to $2.08 \mathrm{~m}$ below mean high water. Coll 1980 by W S Newman, L J Cinquemani, B Duffy, J Schneller, H Greenberg, and K Tessmer.

QC-690. Constitution I. 3

$1440 \pm 100$

2.1 to $2.4 \mathrm{~m}$ below mean high water. Coll 1979 by W S Newman, H Craig, S Jencius, and J Wilson. Comment: sample yields only freshwater diatoms.

QC-695. Constitution I. 4

$2440 \pm 100$

2.9 to $3.4 \mathrm{~m}$ below mean high water. Coll 1979 by W S Newman, H Craig, $\mathrm{S}$ Jencius, and $\mathrm{J}$ Wilson.

QC-226. Constitution I. 5

$2320 \pm 100$

3.7 to $3.9 \mathrm{~m}$ below mean high water. Coll 1976 by L J Cinquemani.

QC-693. Constitution I. 6

$3210 \pm 110$

4.7 to $5.2 \mathrm{~m}$ below mean high water. Coll 1979 by W S Newman, H Craig, $\mathrm{S}$ Jencius, and J Wilson.

QC-1042. Constitution I. 7

$4660 \pm 130$

5.8 to $6.1 \mathrm{~m}$ below mean high water. Coll 1980 by W S Newman, L J Cinquemani, B Duffy, J Schneller, H Greenberg, and K Tessmer.

QC-276. Constitution I. 8

$4110 \pm 100$

5.95 to $6.15 \mathrm{~m}$ below mean high water. Coll 1976 by L J Cinquemani.

QC-694. Constitution I. 9

$3760 \pm 120$

6.1 to $6.6 \mathrm{~m}$ below mean high water. Coll 1979 by W S Newman, H Craig, S Jencius, and J Wilson.

QC-696. Constitution I. 10

$2460 \pm 110$

6.7 to $7.2 \mathrm{~m}$ below mean high water. Coll 1979 by W S Newman, H Craig, S Jencius, and J Wilson. Comment: field notes suggest some sediment flowed into sample.

QC-227. Constitution I. 11

$4230 \pm 120$

7.5 to $7.7 \mathrm{~m}$ below mean high water. Coll 1976 by L J Cinquemani. 
QC-1040. Constitution I. 12

$6030 \pm 290$

7.9 to $8.3 \mathrm{~m}$ below mean high water. Coll 1980 by W S Newman, L J Cinquemani, B Duffy, J Schneller, H Greenberg, and K Tessmer.

QC-189. Constitution I. 13

$\mathbf{5 5 7 0} \pm \mathbf{3 0 0}$

9.25 to $9.45 \mathrm{~m}$ below mean high water. Coll 1976 by L J Cinquemani.

QC-692. Constitution I. 14

$4660 \pm 140$

9.35 to $9.75 \mathrm{~m}$ below mean high water. Coll 1979 by W S Newman, H Craig, S Jencius, and J Wilson. Comment: Marine Transgression appears more pronounced on $\mathrm{E}$ side of marsh.

QC-706. Manitou Marsh

$3530 \pm 110$

Basal peat, 3.65 to $4 \mathrm{~m}$ below mean high water $\left(41^{\circ} 20^{\prime} 00^{\prime \prime} \mathrm{N}, 73^{\circ} 58^{\prime}\right.$ 00" W). Coll 1979 by H Craig, S Jencius, and J Wilson.

\section{Iona Island series}

This series was taken from Ring Meadow, Iona Island. All samples are basal peat except where noted and at coordinates $\left(41^{\circ} 18^{\prime} 00^{\prime \prime} \mathrm{N}, 73^{\circ}\right.$ $\left.58^{\prime} 50^{\prime \prime} \mathrm{W}\right)$.

QC-574. Iona I. 1

$$
390 \pm 100
$$

0.94 to $1.14 \mathrm{~m}$ below mean high water. Coll 1978 by W S Newman.

QC-763. Iona I. 2

$1040 \pm 120$

0.6 to $\mathrm{lm}$ below mean high water. Coll 1979 by H Craig, S Jencius, and F Ciapetti.

QC-764. Iona I. 3

$2240 \pm 120$

Wood and peat, 1.7 to $2 \mathrm{~m}$ below mean high water. Coll 1979 by $\mathrm{H}$ Craig, $S$ Jencius, and F Ciapetti.

QC-575. Iona I. 4

$$
1460 \pm 90
$$

1.98 to $2.18 \mathrm{~m}$ below mean high water. Coll 1978 by W S Newman.

\section{QC-1021. Iona I. 5}

$3430 \pm 120$

2.5 to $2.75 \mathrm{~m}$ below mean high water. Coll 1980 by W S Newman, L J Cinquemani, J Schneller, K Tessmer, and H Greenberg.

QC-765. Iona I. 6

$2140 \pm 100$

2.7 to $3 \mathrm{~m}$ below mean high water. Coll 1979 by H Craig, S Jencius, and F Ciapetti.

QC-576. Iona I. 7

$2830 \pm 130$ Newman.

Wood, 3.24 to $3.44 \mathrm{~m}$ below mean high water. Coll 1978 by W S

QC-1022. Iona I. 8

$3510 \pm 150$

3.41 to $3.71 \mathrm{~m}$ below mean high water. Coll 1980 by W S Newman, L J Cinquemani, J Schneller, K Tessmer, and H Greenberg. 
QC-766. Iona I. 9

$2840 \pm 110$

Peat, 3.6 to $3.9 \mathrm{~m}$ below mean high water. Coll 1979 by H Craig, S Jencius, and F Ciapetti.

QC-1019. Iona I. 10

$4270 \pm 260$

4.37 to $4.67 \mathrm{~m}$ below mean high water. Coll 1980 by W S Newman, L J Cinquemani, J Schneller, K Tessmer, and H Greenberg.

QC-274. Iona I. 11

$3610 \pm 120$

4.4 to $4.6 \mathrm{~m}$ below mean high water. Coll 1976 by L J Cinquemani and W S Newman.

QC-187. Iona I. 12

$3800 \pm 160$

4.55 to $4.75 \mathrm{~m}$ below mean high water. Coll 1976 by L J Cinquemani.

QC-577. Iona I. 13

$4520 \pm 120$

4.89 to $5.09 \mathrm{~m}$ below mean high water. Coll 1978 by W S Newman.

QC-767. Iona I. 14

$3140 \pm 110$

4.9 to $5.3 \mathrm{~m}$ below mean high water. Coll 1979 by H Craig, S Jencius, and F Ciapetti.

QC-1023. Iona I. 15

$4800 \pm 190$

5.6 to $5.85 \mathrm{~m}$ below mean high water. Coll 1980 by W S Newman, L J Cinquemani, J Schneller, K Tessmer, and H Greenberg.

QC-768. Iona I. 16

$2960 \pm 100$

6.1 to $6.5 \mathrm{~m}$ below mean high water. Coll 1979 by H Craig, S Jencius, and F Ciapetti.

QC-1020. Iona I. 17

$4370 \pm 270$

6.25 to $6.55 \mathrm{~m}$ below mean high water. Coll 1980 by W S Newman, L J Cinquemani, J Schneller, K Tessmer, and H Greenberg.

QC-1024. Iona I. 18

$5060 \pm 270$

6.6 to $6.9 \mathrm{~m}$ below mean high water. Coll 1980 by W S Newman, L J Cinquemani, J Schneller, K Tessmer, and H Greenberg.

QC-775. Iona I. 19

$3870 \pm 120$

Peat, 7 to $7.4 \mathrm{~m}$ below mean high water. Coll 1979 by H Craig, S Jencius, and F Ciapetti.

QC-776. Iona I. 20

Peat, 7.6 to $8 \mathrm{~m}$ below mean high water. Coll 1979 by H Craig, S Jencius, and F Ciapetti.

QC-777. Iona I. 21

Peat, 8.1 to $8.5 \mathrm{~m}$ below mean high water. Coll 1979 by $\mathrm{H}$ Craig, S Jencius, and F Ciapetti. 
QC-778. Iona I. 22

$4270 \pm 120$

Peat, 9.6 to $10 \mathrm{~m}$ below mean high water. Coll 1979 by H Craig, S Jencius, and F Ciapetti.

QC-186. Iona I. 23

$3940 \pm 140$

10.55 to $10.75 \mathrm{~m}$ below mean high water. Coll 1976 by L J Cinquemani. General Comment: Iona I. samples 2, 3, 6, 9, 14, 16, 19, 20, 21, 22, and 23 coll along transect $100 \mathrm{~m} \mathrm{~S}$ of other samples and yield considerably higher transgression rates. These samples appear to have been taken from SE block of Timp Fault.

\section{Roa Hook series}

This series was taken from tidal marsh at Roa Hook (Camp Smith). All samples are basal peat, at coordinates $\left(41^{\circ} 17^{\prime} 58^{\prime \prime} \mathrm{N}, 73^{\circ} 56^{\prime} 50^{\prime \prime} \mathrm{W}\right)$; coll 1978 except where noted. Roa Hook 1-6 are W of E-facing buried (fault?) scarp; other samples of this series are E of scarp.

QC-511. Roa Hook 1

$126 \%$ modern

From marsh surface. Coll by W S Newman.

QC-569. Roa Hook 2

$2490 \pm 120$

1.94 to $2.14 \mathrm{~m}$ below mean high water. Coll by W S Newman.

QC-722. Roa Hook 3

$2360 \pm 100$

Wood and basal peat, 2.3 to $2.6 \mathrm{~m}$ below mean high water. Coll 1979 by $\mathrm{H}$ Craig, $\mathrm{S}$ Jencius, and $\mathrm{F}$ Ciapetti.

QC-567. Roa Hook 4

$4280 \pm 110$

3.83 to $4.03 \mathrm{~m}$ below mean high water. Coll by W S Newman. Comment: result suspect because of data item omission.

QC-568. Roa Hook 5

$3170 \pm 170$

4.01 to $4.21 \mathrm{~m}$ below mean high water. Coll by W S Newman.

QC-1041. Roa Hook 6

$3190 \pm 160$

4.25 to $4.55 \mathrm{~m}$ below mean high water $\left(41^{\circ} 17^{\prime} 30^{\prime \prime} \mathrm{N}, 73^{\circ} 56^{\prime} 00^{\prime \prime} \mathrm{W}\right)$. Coll 1980 by W S Newman, L J Cinquemani, B Duffy, J Schneller, H Greenberg, and $\mathrm{K}$ Tessmer.

\section{QC-510. Roa Hook 7}

$3140 \pm 170$

4.8 to $5 \mathrm{~m}$ below mean high water. Coll by W S Newman, R R Pardi, G Greengold, and H Craig.

QC-721. Roa Hook 8

5.5 to $5.8 \mathrm{~m}$ below mean high water. Coll 1979 by H Craig, S Jencius, and F Ciapetti.

QC-723. Roa Hook 9

$3910 \pm 130$

6.7 to $7 \mathrm{~m}$ below mean high water. Coll 1979 by $\mathrm{H}$ Craig, S Jencius, and F Ciapetti. 
QC-566. Roa Hook 10

$4660 \pm 100$ Newman.

Wood and peat, 6.87 to $7.07 \mathrm{~m}$ below mean high water. Coll by W S

QC-1043. Roa Hook 11

$4450 \pm 200$

7.5 to $7.95 \mathrm{~m}$ below mean high water. Coll 1980 by W S Newman, L J Cinquemani, B Duffy, J Schneller, H Greenberg, and K Tessmer.

QC-565. Roa Hook 12

$5470 \pm 140$

Wood and peat, 8.6 to $8.8 \mathrm{~m}$ below mean high water. Coll by W $\mathrm{S}$ Newman.

QC-512. Roa Hook 13

$4120 \pm 350$

8.8 to $9 \mathrm{~m}$ below mean high water. Coll by W S Newman.

QC-509. Roa Hook 14

$4550 \pm 130$

9.3 to $9.5 \mathrm{~m}$ below mean high water. Coll by W S Newman, R R Pardi, G Greengold, and H Craig.

QC-573. Roa Hook 15

$6230 \pm 120$

Wood, 10.8 to $11 \mathrm{~m}$ below mean high water. Coll by W S Newman.

\section{Stony Point series}

This series was taken from tidal marsh $\mathrm{S}$ of Stony Point. All samples are basal peat, at coordinates $\left(41^{\circ} 14^{\prime} 40^{\prime \prime} \mathrm{N}, 73^{\circ} 58^{\prime} 05^{\prime \prime} \mathrm{W}\right)$; coll 1978 by W S Newman, L J Cinquemani, H Craig, S Nelson, and V Newman except where noted.

\section{QC-505. Stony Point 1}

$3100 \pm 110$

3.2 to $3.4 \mathrm{~m}$ below mean high water.

QC-506. Stony Point 2

$3740 \pm 200$

5.8 to $6 \mathrm{~m}$ below mean high water.

QC-469. Stony Point 3

$4830 \pm 110$

5.9 to $6.1 \mathrm{~m}$ below mean high water. Coll 1977 by W S Newman, L J Cinquemani, H Craig, G Greengold, V Newman, and S Nelson.

\section{Oscawana Island series}

This series was taken from Tidal Marsh, Oscawana I. All samples are basal peat, at coordinates $\left(41^{\circ} 13^{\prime} 45^{\prime \prime} \mathrm{N}, 73^{\circ} 55^{\prime} 50^{\prime \prime} \mathrm{W}\right)$; coll 1976 by $\mathrm{L} \mathrm{J}$ Cinquemani except where noted.

\section{QC-228. Oscawana I. 1}

$1870 \pm 90$

2.5 to $2.7 \mathrm{~m}$ below mean high water.

QC-729. Oscawana I. 2

$330 \pm 100$

5.6 to $5.9 \mathrm{~m}$ below mean high water. Coll 1979 by $\mathrm{H}$ Craig and $\mathrm{S}$ Jencius. Comment: Oscawana 2 date seems inexplicable for its depth. 
QC-221B. Oscawana I. 3

$4570 \pm 120$

6.6 to $6.8 \mathrm{~m}$ below mean high water.

QC-264. Oscawana I. 4

$4500 \pm 100$

6.8 to $7 \mathrm{~m}$ below mean high water.

QC-221A. Oscawana I. 5

$5150 \pm 210$

7.3 to $7.5 \mathrm{~m}$ below mean high water.

\section{Cedar Pond series}

This series was taken from Cedar Pond Brook Marsh. All samples are basal peat, at coordinates $\left(41^{\circ} 13^{\prime} 30^{\prime \prime} \mathrm{N}, 73^{\circ} 58^{\prime} 00^{\prime \prime} \mathrm{W}\right)$; coll 1979.

QC-770. Cedar Pond 1

$800 \pm 100$

0.7 to $1 \mathrm{~m}$ below mean high water. Coll by $\mathrm{H}$ Craig, $\mathrm{S}$ Jencius, $\mathrm{F}$ Ciapetti, and H Greenberg.

QC-772. Cedar Pond 2

$1740 \pm 100$

1.7 to $2 \mathrm{~m}$ below mean high water. Coll by $\mathrm{H}$ Craig, $\mathrm{S}$ Jencius, $\mathrm{F}$ Ciapetti, and H Greenberg.

QC-712. Cedar Pond 3

$1940 \pm 110$

2.5 to $2.8 \mathrm{~m}$ below mean high water. Coll by H Craig and S Jencius.

QC-773. Cedar Pond 4

$2650 \pm 100$

2.5 to $2.8 \mathrm{~m}$ below mean high water. Coll by $\mathrm{H}$ Craig, $\mathrm{S}$ Jencius, $\mathrm{F}$ Ciapetti, and $\mathrm{H}$ Greenberg.

QC-771. Cedar Pond 5

$2890 \pm 130$

Wood and peat, 3.1 to $3.4 \mathrm{~m}$ below mean high water. Coll by H Craig, $\mathrm{S}$ Jencius, F Ciapetti, and H Greenberg.

QC-810. Cedar Pond 6

$3030 \pm 100$

3.2 to $3.6 \mathrm{~m}$ below mean high water. Coll by $\mathrm{H}$ Craig, $\mathrm{S}$ Jencius, J Gordon, F Ciapetti, and H Greenberg.

QC-709. Cedar Pond 7

$2220 \pm 120$

3.25 to $3.6 \mathrm{~m}$ below mean high water. Coll by $\mathrm{H}$ Craig, S Jencius, and S Olgun.

QC-774. Cedar Pond 8

$3090 \pm 110$

3.4 to $3.7 \mathrm{~m}$ below mean high water. Coll by $\mathrm{H}$ Craig, S Jencius, $\mathrm{F}$ Ciapetti, and H Greenberg.

QC-811. Cedar Pond 9

$2700 \pm 120$

3.5 to $3.9 \mathrm{~m}$ below mean high water. Coll by $\mathrm{H}$ Craig, $\mathrm{S}$ Jencius, J Gordon, F Ciapetti, and H Greenberg.

QC-710. Cedar Pond 10

$3660 \pm 110$

3.85 to $4.2 \mathrm{~m}$ below mean high water. Coll by $\mathrm{H}$ Craig and $\mathrm{S}$ Jencius. 
QC-812. Cedar Pond 11

$3860 \pm 150$

4.2 to $4.6 \mathrm{~m}$ below mean high water. Coll by $\mathrm{H}$ Craig, S Jencius, J Gordon, F Ciapetti, and H Greenberg.

QC-711. Cedar Pond 12

$3630 \pm 110$

5.1 to $5.5 \mathrm{~m}$ below mean high water. Coll by $\mathrm{H}$ Craig and S Jencius.

QC-718. Cedar Pond 13

$4400 \pm 130$

6.6 to $7 \mathrm{~m}$ below mean high water. Coll by H Craig and S Jencius.

QC-719. Cedar Pond 14

$5080 \pm 130$

6.7 to $7 \mathrm{~m}$ below mean high water. Coll by $\mathrm{H}$ Craig and S Jencius.

\section{Piermont series}

This series was taken from Piermont Tidal Marsh, Tallman State Park. All samples are basal peat, at coordinates $\left(41^{\circ} 01^{\prime} 30^{\prime \prime} \mathrm{N}, 73^{\circ} 54^{\prime} 00^{\prime \prime}\right.$ W); coll 1979 by H Craig and S Jencius except where noted.

\section{QC-733. Piermont 1}

0.7 to $1 \mathrm{~m}$ below mean high water.

\section{QC-734. Piermont 2}

1.4 to $1.7 \mathrm{~m}$ below mean high water.

\section{QC-735. Piermont 3}

$$
2000 \pm 110
$$

3 to $3.3 \mathrm{~m}$ below mean high water.

QC-211. Piermont 4

$$
2300 \pm 160
$$

2.8 to $3 \mathrm{~m}$ below mean high water. Coll 1976 by B Cirolli, M Drillings, J Gordon, and M Balarazo.

\section{QC-736. Piermont 5}

$$
2550 \pm 140
$$

4.5 to $4.8 \mathrm{~m}$ below mean high water.

\section{QC-732. Piermont 6}

$2990 \pm 100$

4.5 to $4.8 \mathrm{~m}$ below mean high water.

\section{QC-730. Piermont 7}

$$
\mathbf{3 0 5 0} \pm \mathbf{1 0 0}
$$

5.2 to $5.5 \mathrm{~m}$ below mean high water.

QC-738. Piermont 8

$$
3320 \pm 140
$$

6.65 to $7 \mathrm{~m}$ below mean high water.

QC-262. Piermont 9

$3460 \pm 100$

4.85 to $5.05 \mathrm{~m}$ below mean high water. Coll by L J Cinquemani.

QC-731. Piermont 10

$3530 \pm 110$

Wood hash, 5.1 to $5.2 \mathrm{~m}$ below mean high water. Comment: samples are not basal peat above Piermont 7 .

QC-737. Piermont 11

$\mathbf{3 7 3 0} \pm \mathbf{2 0 0}$

5.6 to $5.9 \mathrm{~m}$ below mean high water. 
QC-739. Piermont 12

$3790 \pm 90$

7.6 to $8 \mathrm{~m}$ below mean high water.

QC-261. Piermont 13

$4610 \pm 110$

8.34 to $8.54 \mathrm{~m}$ below mean high water. Coll by L J Cinquemani.

QC-740. Piermont 14

$4300 \pm 280$

8.6 to $9 \mathrm{~m}$ below mean sea level.

QC-741. Piermont 15

$4720 \pm 120$

9.6 to $10 \mathrm{~m}$ below mean high water.

QC-742. Piermont 16

$5320 \pm 170$

11.1 to $11.4 \mathrm{~m}$ below mean high water. Coll by $\mathrm{H}$ Craig, S Jencius, and F Ciapetti.

QC-808. Piermont 17

$\mathbf{5 4 8 0} \pm 140$

11 to $11.5 \mathrm{~m}$ below mean high water. Coll by $\mathrm{H}$ Craig, S Jencius, J Gordon, F Ciapetti, and H Greenberg.

QC-809. Piermont 18

$6840 \pm 230$

11.5 to $12 \mathrm{~m}$ below mean high water. Coll by $\mathrm{H}$ Craig, S Jencius, J Gordon, and H Greenberg.

QC-295. Pelham Bay Park

$1800 \pm 90$

Basal peat, 2.05 to $2.25 \mathrm{~m}$ below mean high water $\left(40^{\circ} 52^{\prime} 06^{\prime \prime} \mathrm{N}, 73^{\circ}\right.$ $\left.47^{\prime} 36^{\prime \prime} \mathrm{W}\right)$, Pelham Bay Park, Bronx. Coll 1976 by W S Newman, L J Cinquemani, and $\mathrm{H}$ Craig.

\section{East River series}

This series consists of commercial borehole samples taken from East R. All samples are basal peat, at coordinates $\left(40^{\circ} 47^{\prime} 45^{\prime \prime} \mathrm{N}, 73^{\circ} 49^{\prime} 50^{\prime \prime}\right.$ W) and coll 1976 by Mueser, Rutledge, Johnson, and DeSimone, Consulting Engineers except where noted.

QC-267. College Point Marsh, Core B-206B4, $\quad 5650 \pm 170$ Sample 3

12.7 to $13 \mathrm{~m}$ below mean high water.

QC-306. Roosevelt Ave 1

$7980 \pm 390$

15.5 to $15.7 \mathrm{~m}$ below mean high water $\left(40^{\circ} 48^{\prime} \mathrm{N}, 73^{\circ} 48^{\prime} \mathrm{W}\right)$. Coll by M Marty, Transit Authority.

QC-266. College Point Marsh, Core B-288, Sample 13

$7120 \pm 240$

17.7 to $18 \mathrm{~m}$ below mean high water.

QC-265. College Point Marsh, Core B-219, $\quad 6370 \pm 100$ Sample 15

18.1 to $18.3 \mathrm{~m}$ below mean high water. 
QC-269. College Point Marsh, Core B-227,

$8100 \pm 100$ Sample 15

19.7 to $20.1 \mathrm{~m}$ below mean high water.

QC-268. College Point Marsh, Core B-218, $\quad 12,400 \pm 260$ Sample 13

20 to $20.3 \mathrm{~m}$ below mean high water.

\section{Westway series}

This series was taken along lower W side of Manhattan I. All samples are organic-rich sediments overlying glacial gravels, sands, or rock. Coll 1979 by Mueser, Rutledge, Johnston, and DeSimone, Consulting Engineers except where noted.

QC-1399. Westway MJ0682-0012-057-BVB3 $2700 \pm 150$ $\left.45^{\prime \prime} \mathrm{W}\right)$.

Peat, 1.7 to $1.8 \mathrm{~m}$ below mean high water $\left(40^{\circ} 27^{\prime} 20^{\prime \prime} \mathrm{N}, 74^{\circ} 10^{\prime}\right.$

QC-1381. Westway Core TT-412, Sample $300 \quad 10,700 \pm 180$ 13.7 to $14.3 \mathrm{~m}$ below mean high water $\left(40^{\circ} 45^{\prime} 10^{\prime \prime} \mathrm{N}, 74^{\circ} 00^{\prime} 18^{\prime \prime} \mathrm{W}\right)$.

QC-1382. Westway Core VT-209, Sample 4D $2030 \pm 150$ 18.7 to $19.4 \mathrm{~m}$ below mean high water $\left(40^{\circ} 43^{\prime} 28^{\prime \prime} \mathrm{N}, 74^{\circ} 00^{\prime} 18^{\prime \prime} \mathrm{W}\right)$.

QC-1029. Westway Core HT-1270, Sample $130 D \quad 8190 \pm 130$ Basal peat, 19.2 to $19.8 \mathrm{~m}$ below mean high water $\left(40^{\circ} 43^{\prime} 31^{\prime \prime} \mathrm{N}, 74^{\circ}\right.$ $\left.00^{\prime} 40^{\prime \prime} \mathrm{W}\right)$, from West Side Expressway-Holland Tunnel.

QC-1330. Westway Core VT-249, Sample 8D $490 \pm 110$

Intrusive wood fragment probably from pier piling, 21.3 to $21.9 \mathrm{~m}$ below mean high water $\left(40^{\circ} 43^{\prime} 50^{\prime \prime} \mathrm{N}, 74^{\circ} 00^{\prime} 46^{\prime \prime} \mathrm{W}\right)$.

QC-1380. Westway Core VT-203, Sample $11 D \quad \mathbf{8 9 6 0} \pm \mathbf{2 7 0}$

21.3 to $21.9 \mathrm{~m}$ below mean high water $\left(40^{\circ} 43^{\prime} 28^{\prime \prime} \mathrm{N}, 74^{\circ} 00^{\prime} 56^{\prime \prime} \mathrm{W}\right)$.

QC-1028. Westway Core HV-21, Sample 60D $8750 \pm 170$

Basal peat, 21.3 to $22 \mathrm{~m}$ below mean high water $\left(40^{\circ} 43^{\prime} 23^{\prime \prime} \mathrm{N}, 74^{\circ}\right.$ $\left.00^{\prime} 59^{\prime \prime} \mathrm{W}\right)$, from West Side Expressway-Chambers St.

QC-1389. Westway Core VT-214, Sample 9D $\quad 7650 \pm 190$ 21.5 t o $22 \mathrm{~m}$ below mean high water $\left(40^{\circ} 43^{\prime} 32^{\prime \prime} \mathrm{N}, 74^{\circ} 00^{\prime} 57^{\prime \prime} \mathrm{W}\right)$.

QC-1027. Westway Core HV-13, Sample 11D $10,500 \pm 500$ Basal peat, 22 to $22.6 \mathrm{~m}$ below mean high water $\left(40^{\circ} 43^{\prime} 26^{\prime \prime} \mathrm{N}, 74^{\circ}\right.$ $00^{\prime} 58^{\prime \prime}$ W), from West Side Expressway-Chambers St.

QC-1026. Westway Core HT-120, Sample 13D $9170 \pm 230$

Basal peat, 22.9 to $23.5 \mathrm{~m}$ below mean high water $\left(40^{\circ} 43^{\prime} 34^{\prime \prime} \mathrm{N}, 74^{\circ}\right.$ $00^{\prime} 43^{\prime \prime} \mathrm{W}$ ), from West Side Expressway-Holland Tunnel. 
QC-1184. Westway Core HT-124, Sample 19D $5540 \pm 160$

Shell probably allochthonous, 23.2 to $23.5 \mathrm{~m}$ below mean high water $\left(40^{\circ} 43^{\prime} 33^{\prime \prime} \mathrm{N}, 74^{\circ} 00^{\prime} 40^{\prime \prime} \mathrm{W}\right)$, from along Holland Tunnel, right-of-way on $\mathrm{E}$ side of Hudson R.

QC-1321. Westway Core TT-313, Sample 12D $7920 \pm 200$

24.4 to $25 \mathrm{~m}$ below mean high water $\left(40^{\circ} 44^{\prime} 27^{\prime \prime} \mathrm{N}, 74^{\circ} 00^{\prime} 40^{\prime \prime} \mathrm{W}\right)$.

QC-1374. Westway Core VT-215, Sample 15D $8690 \pm 190$

Organic rich sediment just above bedrock, 24.4 to $25 \mathrm{~m}$ below mean high water $\left(40^{\circ} 43^{\prime} 30^{\prime \prime} \mathrm{N}, 74^{\circ} 00^{\prime} 59^{\prime \prime} \mathrm{W}\right)$.

QC-1025. Westway Core HT-123U, Sample 27D $11,300 \pm 220$

Basal peat, 24.8 to $25.5 \mathrm{~m}$ below mean high water $\left(40^{\circ} 43^{\prime} 32^{\prime \prime} \mathrm{N}, 74^{\circ}\right.$ $\left.00^{\prime} 40^{\prime \prime} \mathrm{W}\right)$, from West Side Expressway-Holland Tunnel.

QC-1324. Westway Core TT-335, Sample 14D 11,100 \pm 250

27.4 to $28 \mathrm{~m}$ below mean high water $\left(40^{\circ} 44^{\prime} 30^{\prime \prime} \mathrm{N}, 74^{\circ} 00^{\prime} 36^{\prime \prime} \mathrm{W}\right)$.

QC-1322. Westway Core TT-314, Sample 15D $11,420 \pm 250$

27.4 to $28 \mathrm{~m}$ below mean high water $\left(40^{\circ} 44^{\prime} 27^{\prime \prime} \mathrm{N}, 74^{\circ} 00^{\prime} 40^{\prime \prime} \mathrm{W}\right)$.

QC-1329. Westway Core TT-359, Sample 16D $11,990 \pm 220$

29 to $29.6 \mathrm{~m}$ below mean high water $\left(40^{\circ} 44^{\prime} 36^{\prime \prime} \mathrm{N}, 74^{\circ} 00^{\prime} 34^{\prime \prime} \mathrm{W}\right)$.

QC-1326. Westway Core TT-352, Sample 17D $11,620 \pm 200$

30.5 to $31.1 \mathrm{~m}$ below mean high water $\left(40^{\circ} 44^{\prime} 35^{\prime \prime} \mathrm{N}, 74^{\circ} 00^{\prime} 36^{\prime \prime} \mathrm{W}\right)$.

QC-1183. Westway Core RR-114, Sample 22D $9540 \pm 120$

Organic silt, 36.6 to $37.2 \mathrm{~m}$ below mean high water $\left(40^{\circ} 45^{\prime} 39^{\prime \prime} \mathrm{N}\right.$, $\left.74^{\circ} 00^{\prime} 47^{\prime \prime} \mathrm{W}\right)$, from Amtrak Tunnel-E shore Hudson R. Comment: not basal peat.

QC-1182. Westway Core RR-126, Sample 27 10,200 \pm 170

Wood fragments overlying glacial gravel, 38.2 to $38.9 \mathrm{~m}$ below mean high water $\left(40^{\circ} 45^{\prime} 36^{\prime \prime} \mathrm{N}, 74^{\circ} 00^{\prime} 45^{\prime \prime} \mathrm{W}\right)$; coll 1978. Comment: probably allochonthonous sample.

QC-1315. Westway Core WT-505, Sample 26D $12,280 \pm 260$

Lowest organic material above glacial gravel, 42.7 to $43.3 \mathrm{~m}$ below mean high water $\left(40^{\circ} 45^{\prime} 35^{\prime \prime} \mathrm{N}, 74^{\circ} 00^{\prime} 45^{\prime \prime} \mathrm{W}\right)$.

\section{Caumsett Marsh series}

This series was taken from Caumsett Marsh. All samples are basal peat, at coordinates $\left(40^{\circ} 56^{\prime} 30^{\prime \prime} \mathrm{N}, 73^{\circ} 28^{\prime} 50^{\prime \prime} \mathrm{W}\right)$; coll 1979 by D Habib, W S Newman, L J Cinquemani, H Craig, S Jencius, and J Wilson.

QC-689. Caumsett Marsh 1

0.9 to $1.1 \mathrm{~m}$ below mean high water.

$780 \pm 120$ 
QC-687. Caumsett Marsh 2

$660 \pm 120$

2.05 to $2.35 \mathrm{~m}$ below mean high water.

QC-688. Caumsett Marsh 3

$760 \pm 140$

2.06 to $2.36 \mathrm{~m}$ below mean high water.

\section{Eatons Neck series}

This series are all basal peats from tidal marsh on Eatons Neck, at coordinates $\left(40^{\circ} 56^{\prime} 58^{\prime \prime} \mathrm{N}, 73^{\circ} 23^{\prime} 43^{\prime \prime} \mathrm{W}\right)$. Coll 1979 by G Wisker.

QC-681. Eatons Neck 1

$370 \pm 120$

0.65 to $0.95 \mathrm{~m}$ below mean high water.

QC-679. Eatons Neck 2

$1590 \pm 11$

1.4 to $1.55 \mathrm{~m}$ below mean high water.

QC-682. Eatons Neck 3

$2520 \pm 90$

4.85 to $5.05 \mathrm{~m}$ below mean high water.

QC-190. Mt Sinai

$2180 \pm 100$

Peat, 4.2 to $4.9 \mathrm{~m}$ below surface $\left(40^{\circ} 56^{\prime} 55^{\prime \prime} \mathrm{N}, 73^{\circ} 01^{\prime} 50^{\prime \prime} \mathrm{W}\right)$, from Mt Sinai Harbor. Coll 1976 by L J Cinquemani.

\section{Shelter Island series}

This series was taken from Bass Creek, Shelter I. All samples are at coordinates $\left(41^{\circ} 02^{\prime} 47^{\prime \prime} \mathrm{N}, 72^{\circ} 18^{\prime} 50^{\prime \prime} \mathrm{W}\right)$; coll 1980 by W $\mathrm{S}$ Newman, M Newman, B Duffy, L Bruno, and J Isby.

QC-1084. Shelter I. 1

$850 \pm 150$

Salt marsh-sphagnum peat interface, 1.1 to $1.3 \mathrm{~m}$ below mean high water.

QC-1083A\&B. Shelter I. 2

$3590 \pm 130$

Base of sphagnum peat sec above glacial drift. Peat and wood, 6.6 to $6.75 \mathrm{~m}$ below mean high water.

QC-1082. Queens Mall, Core 5, Sample 14B

Peat, 22.8 to $23.5 \mathrm{~m}$ below mean high water $\left(40^{\circ} 44^{\prime} 05^{\prime \prime} \mathrm{N}, 73^{\circ} 52^{\prime} 30^{\prime \prime}\right.$ W), from Queens Mall, Rego Park, near intersec of Queens Blvd and Woodhaven Blvd. Coll 1973 by Woodward Moorehouse Assoc. Comment: sample in Flushing Formation (see Newman, 1977).

\section{New Jersey}

\section{Cheesequake series}

This series was taken from salt marsh in Cheesequake State Park. All samples are basal peat except Cheesequake 6, at coordinates $\left(40^{\circ} 26^{\prime} 05^{\prime \prime}\right.$ $\mathrm{N}, 74^{\circ} 17^{\prime} 20^{\prime \prime} \mathrm{W}$ ); coll 1979 by W S Newman, H Craig, S Jencius, H Greenberg, and R Ortner except where noted. Cheesequake 4 through 6 appear anomalous in age and/or elev. 
QC-844. Cheesequake 1

$1210 \pm 190$

2.6 to $2.8 \mathrm{~m}$ below mean high water.

QC-847. Cheesequake 2

$1960 \pm 130$

2.8 to $3.05 \mathrm{~m}$ below mean high water.

QC-842. Cheesequake 3

$2080 \pm 160$

3.3 to $3.5 \mathrm{~m}$ below mean high water.

QC-848. Cheesequake 4

$930 \pm 170$

4.5 to $4.8 \mathrm{~m}$ below mean high water.

QC-846. Cheesequake 5

$\mathbf{5 3 0} \pm \mathbf{1 5 0}$

9.25 to $9.65 \mathrm{~m}$ below mean high water.

QC-845. Cheesequake 6

$4820 \pm 100$

Peat, 10.9 to $11.15 \mathrm{~m}$ below mean high water.

QC-896. Cheesequake $7 \quad \mathbf{7 2 3 0} \pm 190$

11.8 to $12.1 \mathrm{~m}$ below mean high water. Coll by $\mathrm{H}$ Craig, S Jencius, $\mathrm{H}$ Greenberg, and $\mathrm{K}$ Tessmer.

\section{Sea Island City series}

This series was taken from Sea Island City. All samples are basal peat and coll 1979 by W S Newman, H Craig, S Jencius, H Greenberg, and R Ortner.

QC-849. Sea I. City 1

0.7 to $0.9 \mathrm{~m}$ below mean high water $\left(39^{\circ} 10^{\prime} 40^{\prime \prime} \mathrm{N}, 74^{\circ} 43^{\prime} 45^{\prime \prime} \mathrm{W}\right)$. Comment: modern rootlet contamination.

QC-850. Sea I. City 2

$920 \pm 160$

1.3 to $1.5 \mathrm{~m}$ below mean high water $\left(39^{\circ} 10^{\prime} 30^{\prime \prime} \mathrm{N}, 74^{\circ} 43^{\prime} 35^{\prime \prime} \mathrm{W}\right)$.

QC-851. Sea I. City 3

$2350 \pm 100$ $\left.25^{\prime \prime} \mathrm{W}\right)$.

From layer 2.8 to $3 \mathrm{~m}$ below mean high water $\left(39^{\circ} 10^{\prime} 15^{\prime \prime} \mathrm{N}, 74^{\circ} 43^{\prime}\right.$

QC-852. Sea I. City 4

$2260 \pm 100$

3.5 to $3.7 \mathrm{~m}$ below mean high water $\left(39^{\circ} 10^{\prime} 00^{\prime \prime} \mathrm{N}, 74^{\circ} 43^{\prime} 15^{\prime \prime} \mathrm{W}\right)$.

QC-853. Sea I. City 5

$2760 \pm 100$

4.75 to $4.95 \mathrm{~m}$ below mean high water $\left(39^{\circ} 09^{\prime} 45^{\prime \prime} \mathrm{N}, 74^{\circ} 43^{\prime} 05^{\prime \prime} \mathrm{W}\right)$.

QC-854. Sea I. City 6

$3440 \pm 110$

5.45 to $5.75 \mathrm{~m}$ below mean high water $\left(39^{\circ} 09^{\prime} 40^{\prime \prime} \mathrm{N}, 74^{\circ} 42^{\prime} 45^{\prime \prime} \mathrm{W}\right)$.

QC-855. Sea I. City 7

$3960 \pm 110$

7.3 to $7.6 \mathrm{~m}$ below mean high water $\left(39^{\circ} 09^{\prime} 30^{\prime \prime} \mathrm{N}, 74^{\circ} 42^{\prime} 25^{\prime \prime} \mathrm{W}\right)$. 
Connecticut

\section{Indian River series}

This series was taken from Indian River, Milford. All samples are basal peat at coordinates $\left(41^{\circ} 13^{\prime} 10^{\prime \prime} \mathrm{N}, 73^{\circ} 02^{\prime} 12^{\prime \prime} \mathrm{W}\right)$; coll 1980 by $\mathrm{W}$ S Newman, H Greenberg, L J Cinquemani, K Tessmer, J Schneller, and W Krulish.

\section{QC-1017A\&B. Indian R 1}

$$
2970 \pm 100
$$

3.2 to $3.65 \mathrm{~m}$ below mean high water.

\section{QC-1012A\&B. Indian R 2}

$3500 \pm 120$

4.25 to $4.45 \mathrm{~m}$ below mean high water.

QC-1010A\&B. Indian R 3

$$
3650 \pm 100
$$

5.3 to $5.7 \mathrm{~m}$ below mean high water.

QC-1016. Gulf Pond

$1520 \pm 190$

Basal peat, 2 to $2.2 \mathrm{~m}$ below mean high water $\left(41^{\circ} 13^{\prime} 00^{\prime \prime} \mathrm{N}, 73^{\circ} 12^{\prime}\right.$ $00^{\prime \prime}$ W), from Gulf Pond, Milford. Coll 1980 by W S Newman, L J Cinquemani, H Greenberg, K Tessmer, J Schneller, and W Krulish.

\section{Oyster Creek series}

This series was taken from Oyster Creek, Old Saybrook. All samples are basal peat at coordinates $\left(41^{\circ} 15^{\prime} 20^{\prime \prime} \mathrm{N}, 72^{\circ} 21^{\prime} 00^{\prime \prime} \mathrm{W}\right)$; coll 1980 by W S Newman, L J Cinquemani, H Greenberg, K Tessmer, J Schneller, and W Krulish.

QC-1015A. Oyster Creek 1

$3970 \pm 390$

3.87 to $3.97 \mathrm{~m}$ below mean high water.

QC-1014. Oyster Creek 2

$4460 \pm 160$

6.53 to $6.83 \mathrm{~m}$ below mean high water.

QC-1014B\&C. Oyster Creek 3

$3850 \pm 240$

6.42 to $6.83 \mathrm{~m}$ below mean high water.

QC-1013. Oyster Creek 4

$4780 \pm 180$

6.95 to $7.15 \mathrm{~m}$ below mean high water.

QC-1011. Oyster Creek 5

$5510 \pm 130$

Shell in basal peat, 7.9 to $8.05 \mathrm{~m}$ below mean high water.

\section{Delaware}

\section{QC-807. Fowler Beach}

Basal peat, 0.4 to $0.55 \mathrm{~m}$ below mean high water $\left(38^{\circ} 53^{\prime} 00^{\prime \prime} \mathrm{N}, 74^{\circ}\right.$

$16^{\prime} 18^{\prime \prime}$ W), from Fowler Beach. Coll 1979 by W S Newman, H Craig, S Jencius, J Gordon, and H Greenberg. 
Maryland

\section{Radcliffe Creek series}

This series was taken from Radcliffe Creek Bridge. All samples are basal peat at coordinates $\left(59^{\circ} 12^{\prime} 00^{\prime \prime} \mathrm{N}, 76^{\circ} 04^{\prime} 00^{\prime \prime} \mathrm{W}\right)$; coll 1979 by W $S$ Newman, H Craig, S Jencius, H Greenberg, R Ortner, and G G Connally.

\section{QC-859. Radeliffe Creek 1}

$1230 \pm 160$

1.8 to $2 \mathrm{~m}$ below mean high water.

QC-857. Radeliffe Creek 2

$3370 \pm 150$

Wood, small acorn, and basal peat, 5 to $5.3 \mathrm{~m}$ below mean high water.

QC-856. Radeliffe Creek 3

$4510 \pm 120$

10.7 to $11 \mathrm{~m}$ below mean high water.

\section{Blackwater series}

This series was taken from Blackwater Wildlife Refuge. All samples coll 1979 by W S Newman, H Craig, S Jencius, H Greenberg, and R Ortner.

QC-860. Blackwater 1

Basal peat and some wood hash, 3.2 to $3.45 \mathrm{~m}$ below mean high water $\left(38^{\circ} 23^{\prime} 32^{\prime \prime} \mathrm{N}, 76^{\circ} 03^{\prime} 45^{\prime \prime} \mathrm{W}\right)$.

\section{QC-861. Blackwater 2}

$2490 \pm 130$

Basal peat and some wood, 3.5 to $3.7 \mathrm{~m}$ below mean high water $\left(38^{\circ}\right.$ $23^{\prime} 23^{\prime \prime} \mathrm{N}, 76^{\circ} 03^{\prime} 50^{\prime \prime} \mathrm{W}$ ).

\section{QC-862. Blackwater 3} $\left.55^{\prime \prime} \mathrm{W}\right)$.

Basal peat, 4 to $4.2 \mathrm{~m}$ below mean high water $\left(38^{\circ} 23^{\prime} 15^{\prime \prime} \mathrm{N}, 76^{\circ} 03^{\prime}\right.$

\section{QC-863. Blackwater 4}

Basal peat and wood hash, 5.4 to $5.7 \mathrm{~m}$ below mean high water $\left(38^{\circ}\right.$ $23^{\prime} 00^{\prime \prime} \mathrm{N}, 76^{\circ} 04^{\prime} 00^{\prime \prime} \mathrm{W}$ ).

\section{North Carolina}

\section{Roanoke Island series}

This series was taken from Baumtown, Roanoke I. All samples are basal peat at coordinates ( $\left.35^{\circ} 52^{\prime} 30^{\prime \prime} \mathrm{N}, 75^{\circ} 39^{\prime} 00^{\prime \prime} \mathrm{W}\right)$; coll 1979 by W S Newman, H Craig, S Jencius, J Gordon, and H Greenberg.

QC-792. Roanoke I. 1

$$
760 \pm 140
$$

0.4 to $0.6 \mathrm{~m}$ below mean high water.

QC-805. Roanoke I. 2

$2950 \pm 280$

1.1 to $1.3 \mathrm{~m}$ below mean high water.

QC-804. Roanoke I. 3

$2630 \pm 150$

1.4 to $1.7 \mathrm{~m}$ below mean high water. 


\section{Croatan National Forest series}

This series was taken from Croatan Natl Forest. All samples are basal peat at coordinates $\left(34^{\circ} 42^{\prime} \mathrm{N}, 77^{\circ} 06^{\prime} \mathrm{W}\right)$; coll 1979 by W S Newman, $\mathrm{H}$ Craig, S Jencius, J Gordon, and H Greenberg.

QC-801. Croatan Forest 1
0.4 to $0.7 \mathrm{~m}$ below mean high water.

QC-802. Croatan Forest 2

$1180 \pm 190$

1.6 to $1.9 \mathrm{~m}$ below mean high water.

\section{Lilliput Creek series}

This series was taken from Lilliput Creek, Rte 133 near Wilmington. All samples are at coordinates (34 $\left.04^{\prime} 30^{\prime \prime} \mathrm{N}, 77^{\circ} 57^{\prime} 27^{\prime \prime} \mathrm{W}\right)$; coll 1979 by W S Newman, H Craig, S Jencius, J Gordon, and H Greenberg.

QC-798. Lilliput Creek 1

$1450 \pm 150$

Basal peat, 0.6 to $0.8 \mathrm{~m}$ below mean high water.

QC-799. Lilliput Creek 2

$1390 \pm 130$

Basal peat, 1.25 to $1.45 \mathrm{~m}$ below mean high water.

QC-793A. Lilliput Creek 3

$3390 \pm 110$

Wood, 3.1 to $3.3 \mathrm{~m}$ below mean high water.

QC-793B. Lilliput Creek 4

$3400 \pm 110$

Basal peat, 3.5 to $3.8 \mathrm{~m}$ below mean high water.

QC-794. Lilliput Creek 5

$3600 \pm 120$

Wood and peat, 4.2 to $4.5 \mathrm{~m}$ below mean high water.

QC-795. Lilliput Creek 6

$3260 \pm 190$

Basal peat, 4.5 to $4.9 \mathrm{~m}$ below mean high water.

QC-796. Lilliput Creek 7

$3870 \pm 180$

Basal peat, 5.45 to $5.95 \mathrm{~m}$ below mean high water.

QC-797. Lilliput Creek 8

$\mathbf{5 6 8 0} \pm \mathbf{2 5 0}$

Wood and basal peat, 8.1 to $8.36 \mathrm{~m}$ below mean high water.

South Carolina

Pee Dee River series

QC-603. Pee Dee R $1 \quad 2630 \pm 110$

Woody peat, 2.6 to $2.8 \mathrm{~m}$ below mean high water. Coll 1978 by W S Newman and F Stapor.

QC-602. Pee Dee R 2

$3690 \pm 150$

Basal peat, 3.4 to $3.6 \mathrm{~m}$ below mean high water. Coll 1978 by W S Newman and F Stapor.

QC-815. Pee Dee R 3

$\mathbf{5 3 0 0} \pm \mathbf{1 5 0}$

Wood, in basal peat, 3.5 to $3.9 \mathrm{~m}$ below mean high water. Coll 1979 by W S Newman, H Craig, S Jencius, J Gordon, and H Greenberg. 
QC-604. Pee Dee R 4

Wood, 4.8 to $5 \mathrm{~m}$ below mean high water. Coll 1978 by W S Newman and F Stapor.

QC-813. Pee Dee R 5

$5630 \pm 130$

Peat, 6.58 to $6.8 \mathrm{~m}$ below mean high water, $0.3 \mathrm{~m}$ above sand stratum. Coll 1979 by W S Newman, H Craig, S Jencius, J Gordon, and H Greenberg.

QC-814. Pee Dee R 6

$6140 \pm 200$

Basal peat, 6.65 to $7.1 \mathrm{~m}$ below mean high water. Coll 1979 by W $\mathrm{S}$ Newman, H Craig, S Jencius, J Gordon, and H Greenberg.

\section{Santee River series}

This series was taken from Santee River Estuary near Rte 17. All samples are at coordinates $\left(33^{\circ} 11^{\prime} 36^{\prime \prime} \mathrm{N}, 79^{\circ} 23^{\prime} 48^{\prime \prime} \mathrm{W}\right)$; coll 1978 by W S Newman and F Stapor.

QC-596-1. Santee R 1

$3110 \pm 90$

Basal peat, 3 to $3.2 \mathrm{~m}$ below mean high water.

QC-596-2. Santee R 2

$3140 \pm 140$ R 1 .

Basal peat, 3 to $3.2 \mathrm{~m}$ below mean high water. Repeat assay of Santee

QC-597. Santee R 3

$4550 \pm 150$

Wood and peat, 3.87 to $4.15 \mathrm{~m}$ below mean high water.

QC-595. Santee R 4

$4420 \pm 410$

Basal peat, 4.05 to $4.35 \mathrm{~m}$ below mean high water.

QC-598. Santee R 5

$3980 \pm 280$

Wood in paleosol, $5.3 \mathrm{~m}$ below mean high water.

\section{Cooper River series}

This series was taken from Cooper River Estuary. All samples are basal peat and coll 1978 by W S Newman and D J Colquhoun except where noted.

QC-583. Cooper R 1

$$
2040 \pm 110
$$

Wood stump in basal peat on Cooper Marl, $1 \mathrm{~m}$ below mean high water $\left(32^{\circ} 55^{\prime} 30^{\prime \prime} \mathrm{N}, 79^{\circ} 53^{\prime} 42^{\prime \prime} \mathrm{W}\right)$.

QC-611. Cooper R 2

1.6 to $1.8 \mathrm{~m}$ below mean high water $\left(32^{\circ} 58^{\prime} 30^{\prime \prime} \mathrm{N}, 79^{\circ} 54^{\prime} 05^{\prime \prime} \mathrm{W}\right)$.

QC-585. Cooper R 3

$2700 \pm 120$

Stump in basal peat on Cooper Marl, $2 \mathrm{~m}$ below mean high water $\left(32^{\circ}\right.$ $58^{\prime} 31^{\prime \prime} \mathrm{N}, 79^{\circ} 53^{\prime} 40^{\prime \prime} \mathrm{W}$ ). 
QC-613. Cooper R 4

$2330 \pm 140$

I.6 to $2.3 \mathrm{~m}$ below mean high water $\left(32^{\circ} 58^{\prime} 30^{\prime \prime} \mathrm{N}, 79^{\circ} 54^{\prime} 05^{\prime \prime} \mathrm{W}\right)$.

QC-584. Cooper R 5

$3100 \pm 100$

2.5 to $2.7 \mathrm{~m}$ below mean high water $\left(32^{\circ} 58^{\prime} 30^{\prime \prime} \mathrm{N}, 79^{\circ} 54^{\prime} 05^{\prime \prime} \mathrm{W}\right)$.

QC-588. Cooper R 6

$4140 \pm 70$

2.8 to $3.1 \mathrm{~m}$ below mean high water $\left(32^{\circ} 59^{\prime} 50^{\prime \prime} \mathrm{N}, 79^{\circ} 53^{\prime} 52^{\prime \prime} \mathrm{W}\right)$.

QC-587. Cooper R 7

$4290 \pm 130$

3.4 to $3.7 \mathrm{~m}$ below mean high water $\left(32^{\circ} 59^{\prime} 05^{\prime \prime} \mathrm{N}, 79^{\circ} 54^{\prime} 00^{\prime \prime} \mathrm{W}\right)$.

QC-586. Cooper $\mathbf{R} 8$

$5010 \pm 140$

4.3 to $4.7 \mathrm{~m}$ below mean high water $\left(32^{\circ} 58^{\prime} 45^{\prime \prime} \mathrm{N}, 79^{\circ} 53^{\prime} 37^{\prime \prime} \mathrm{W}\right)$.

\section{Wando River series}

This series was taken from Hobcaw Creek, Wando River. All samples are at coordinates $\left(32^{\circ} 48^{\prime} 00^{\prime \prime} \mathrm{N}, 79^{\circ} 53^{\prime} 00^{\prime \prime} \mathrm{W}\right)$ and coll 1979 by $\mathrm{L} \mathrm{J}$ Cinquemani and $\mathrm{H}$ Craig.

QC-703. Wando R 1

$3100 \pm 160$

Basal peat, 2 to $2.2 \mathrm{~m}$ below mean high water.

QC-702. Wando R 2

$4670 \pm 130$

Woody basal peat, 2.75 to $3.05 \mathrm{~m}$ below mean high water.

QC-704. Wando R 3

$4760 \pm 290$

Basal peat and wood hash, 3.9 to $4.2 \mathrm{~m}$ below mean high water.

\section{Combahee River series}

This series was taken from Combahee River. All samples are peat at coordinates $\left(32^{\circ} 39^{\prime} 12^{\prime \prime} \mathrm{N}, 80^{\circ} 40^{\prime} 30^{\prime \prime} \mathrm{W}\right)$; coll 1978 by W S Newman and F W Stapor except where noted.

\section{QC-609. Combahee R 1}

$2880 \pm 110$

2.26 to $2.45 \mathrm{~m}$ below mean high water.

QC-610. Combahee R 2

$3330 \pm 130$

Woody peat, 2.75 to $2.9 \mathrm{~m}$ below mean high water.

QC-828. Combahee R 3

$4430 \pm 170$

Basal peat, 3.32 to $3.66 \mathrm{~m}$ below mean high water. Coll 1979 by W S Newman, H Craig, S Jencius, J Gordon, and H Greenberg.

QC-594. Combahee R 4

$5620 \pm 140$

3.65 to $3.8 \mathrm{~m}$ below mean high water.

QC-593. Combahee R 5

$5280 \pm 120$

4 to $4.2 \mathrm{~m}$ below mean high water.

QC-589. Combahee R 6

$5400 \pm 120$

4.15 to $4.35 \mathrm{~m}$ below mean high water $\left(32^{\circ} 39^{\prime} 20^{\prime \prime} \mathrm{N}, 80^{\circ} 40^{\prime} 14^{\prime \prime} \mathrm{W}\right)$. 


\section{Coosawatchie River series}

This series was taken from Coosawatchie River. All samples are basal peat at coordinates $\left(32^{\circ} 35^{\prime} 00^{\prime \prime} \mathrm{N}, 80^{\circ} 55^{\prime} 15^{\prime \prime} \mathrm{W}\right)$; coll 1979 by D Colquhoun, W S Newman, H Craig, S Jencius, J Gordon, and H Greenberg.

\section{QC-827. Coosawatchie R 1}

$730 \pm 110$

0.7 to $1 \mathrm{~m}$ below mean high water.

\section{QC-826. Coosawatchie R 2}

$2130 \pm 100$

1.28 to $1.58 \mathrm{~m}$ below mean high water.

\section{Savannah River series}

This series was taken from Savannah River Estuary. All samples are at coordinates $\left(32^{\circ} 08^{\prime} 00^{\prime \prime} \mathrm{N}, 80^{\circ} 59^{\prime} 30^{\prime \prime} \mathrm{W}\right)$; coll 1978 by F Stapor and W S Newman except where noted.

QC-825. Savannah R 1

$3130 \pm 130$

Basal peat and some wood, 2 to $2.3 \mathrm{~m}$ below mean high water. Coll 1979 by W S Newman, H Craig, S Jencius, J Gordon, and H Greenberg.

QC-600. Savannah R 2

$2320 \pm 110$

Peat and some wood, 2.5 to $2.7 \mathrm{~m}$ below mean high water.

QC-599. Savannah R 3

$3100 \pm 100$

Basal peat, 2.7 to $2.9 \mathrm{~m}$ below mean high water.

QC-821. Savannah R 4

Basal peat, 3.35 to $3.53 \mathrm{~m}$ below mean high water. Coll 1979 by W S Newman, H Craig, S Jencius, J Gordon, and H Greenberg.

QC-601. Savannah R 5

Peat and roots in paleosol, $4 \mathrm{~m}$ below mean high water.

QC-822. Savannah R 6

Basal peat, 4.77 to $5.07 \mathrm{~m}$ below mean high water. Coll 1979 by W Newman, H Craig, S Jencius, J Gordon, and H Greenberg.

\section{REFFRENCES}

Newman, W S, 1977, Late Quaternary paleoenvironmental reconstruction; some contradictions from northwestern Long Island, in Newman, W S and Salwen, Bert, eds, Amerinds and their paleoenvironments in northeastern North America: Annals New York Acad Sci, v 288, p 545-570.

Pardi, R, and Newman, E R, 1980, Qucens College radiocarbon measurements III: Radiocarbon, v 22, p 1073-1083. 